\title{
Comparison of Clinical Characteristics of Fluorescence in Quantitative Light-Induced Fluorescence Images according to the Maturation Level of Dental Plaque
}

\author{
Eun-Ha Jung ${ }^{1}$ and Hye-Young $\mathrm{Oh}^{2, \dagger}$ \\ 'Department of Dental Hygiene, Yonsei University, Wonju 26493, ${ }^{2}$ Department of Dental Hygiene, Suwon \\ Science College, Hwaseong 18516, Korea
}

\begin{abstract}
Background: Proper detection and management of dental plaque are essential for individual oral health. We aimed to evaluate the maturation level of dental plaque using a two-tone disclosing agent and to compare it with the fluorescence of dental plaque on the quantitative light-induced fluorescence (QLF) image to obtain primary data for the development of a new dental plaque scoring system.

Methods: Twenty-eight subjects who consented to participate after understanding the purpose of the study were screened. The images of the anterior teeth were obtained using the QLF device. Subsequently, dental plaque was stained with a two-tone disclosing solution and a photograph was obtained with a digital single-lens reflex (DSLR) camera. The staining scores were assigned as follows: 0 for no staining, 1 for pink staining, and 2 for blue staining. The marked points on the DSLR images were selected for RGB color analysis. The relationship between dental plaque maturation and the red/green (R/G) ratio was evaluated using Spearman's rank correlation. Additionally, different red fluorescence values according to dental plaque accumulation were assessed using one-way analysis of variance followed by Scheffe's post-hoc test to identify statistically significant differences between the groups.

Results: A comparison of the intensity of red fluorescence according to the maturation of the two-tone stained dental plaque confirmed that R/G ratio was higher in the QLF images with dental plaque maturation $(p<0.001)$. Correlation analysis between the stained dental plaque and the red fluorescence intensity in the QLF image confirmed an excellent positive correlation $(p<0.001)$. Conclusion: A new plaque scoring system can be developed based on the results of the present study. In addition, these study results may also help in dental plaque management in the clinical setting.
\end{abstract}

Key Words: Dental plaque, Dental plaque index, Quantitative light-induced fluorescence, Red fluorescence

\section{Introduction}

Dental plaque is a significant cause of two major oral diseases, namely dental caries and periodontal disease ${ }^{1)}$. Particularly, gingivitis can develop in a short period if dental plaque is not properly managed in the oral cavity ${ }^{2,3)}$. In addition, dental plaque accumulation in the oral cavity for a long time can lead to proliferation of anaerobic bacteria and worsen the gingival inflammatory condition. However, in gingivitis, self-management alone can restore the gingiva to a healthy state. Hence, dental plaque management is extremely important $t^{4)}$.

Plaque indices are generally used to assess patients' oral hygiene and oral self-care status. Currently, plaque indices such as the O'Leary index, PHP index, and Löe and Silness index are available. These are used to detect dental plaque and to manage the oral hygiene status according to their respective purposes ${ }^{5,6)}$. Before dental plaque evaluation, the tooth surface is stained using a disclosing agent to visualize plaque accumulation. The disclosing agents are 
mainly solution-type, gel-type, or tablet-type agents. These are divided into one-tone disclosing agents and two-tone disclosing agents ${ }^{7}$. Currently, a two-tone disclosing solution is frequently used in clinical practice. The advantage of a two-tone solution is that it stains dental plaque with two different colors according to plaque maturation (young plaque is stained pink and old or matured plaque is stained in a bluish tone $)^{8,9)}$. However, the need to perform a separate staining process to detect dental plaque and the need to remove the disclosing agent remaining over the tooth surface after the examination lead to discomfort and extension of the treatment time. Additionally, apoptosis and cytotoxicity due to the use of disclosing agents have been reported in a recently published article. Thus, it is necessary to develop alternative measures ${ }^{10)}$.

Dental plaque detection using a quantitative light-induced fluorescence (QLF) image has been proposed as an alternative to the dental plaque inspection methods using disclosing solutions and to relieve discomfort ${ }^{11}$. The QLF technology involves use of an optical device that uses visible light at $405 \mathrm{~nm}$ to detect porphyrin, a bacterial metabolite in the oral cavity, with red fluorescence ${ }^{12,13)}$. Reasonably, the QLF technology is used to classify the maturation level of dental plaque, since previous studies have confirmed that stronger red fluorescence is associated with dental plaque that has been accumulated for a long time and plaque with a higher pathogenicity ${ }^{14}$. In addition, a QLF image shows the simple plaque score (SPS) using a dedicated software, which makes it easier to recognize the current state of dental plaque with a high degree of agreement with the existing dental surface staining methods, highlighting its potential as a new dental plaque detection method $^{15,16)}$. Although dental plaque detection has many advantages using the QLF technology, it is expensive and time-consuming to obtain objective information by applying dedicated software in a busy clinical setting. In addition, the distribution of dental plaque and red fluorescence in the QLF images is presented as average values in case of SPS. Therefore, even if a specific area is not well managed, good results might be obtained if there is no dental plaque in other areas. Thus, poor oral hygiene can be misdiagnosed as a good oral hygiene status. Therefore, a new dental plaque scoring system is required to overcome these shortcomings.

To develop a new dental plaque scoring system that can classify the depth of the dental plaque on the QLF image, it is necessary to compare the pattern of dental plaque stained using the disclosing solution with the surface of dental plaque observed in the QLF image. However, studies conducted to date have compared the quantitative QLF values and the presence or absence of pigmented dental plaque using a one-tone disclosing solution. Therefore, there was a limitation in comparing the maturation level and pathogenicity of dental plaque observed in the QLF image. As a first step in developing an objective and intuitive dental plaque scoring system, it is necessary to classify the degree of dental plaque maturation in the oral cavity and to compare it with the fluorescence expression pattern in the QLF image.

The present study aimed to provide primary data for developing a new dental plaque index by comparing dental plaque maturation level obtained using a two-tone disclosing agent and the fluorescence of dental plaque captured by the QLF technology.

\section{Materials and Methods}

\section{Participants}

This study was conducted from August 2017 to June 2018. The minimum required sample size was computed

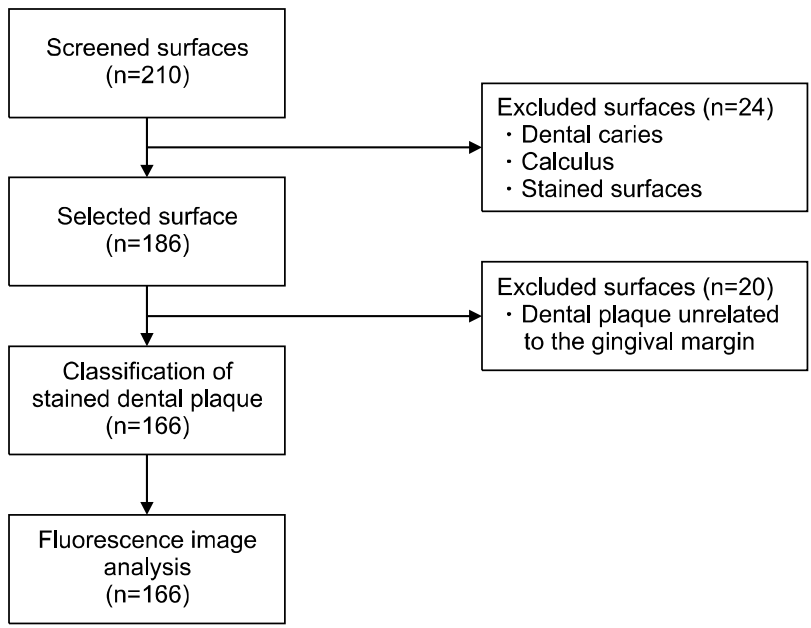

Fig. 1. Flowchart of the study process. $\mathrm{n}$ : number of labial surfaces. 
using the $\mathrm{G}^{*}$ Power program (version 3.1.9.6.; Heinrich Heine Universität Düsseldorf, Düsseldorf, Germany). Considering a significance level $(\alpha)$ of 0.05 , power $(1-\beta)$ of 0.8 , and effect size of 0.5 , the computed minimum sample size was 159 . However, we increased the sample size by $10 \%$ and considered 174 surfaces. The subjects were enrolled according to the inclusion and exclusion criteria of this study. Subjects who consented to participate after understanding the purpose of the study were screened according to the declaration of Helsinki. Finally, 28 participants were selected after screening dental images of 35 participants (Fig. 1).

\section{Method}

\section{1) Obtaining the test images}

The incisor teeth were taken using the Q-ray cam (AIOBIO, Seoul, Korea) and a digital single-lens reflex (DSLR) camera (Nikon D, Tokyo, Japan). To capture the fluorescence images, the Q-ray cam was connected to a desktop computer and the cheek was retracted using a cheek retractor. The Q-ray cam imaging used the following parameters: resolution (image size), full high-definition $(1,920 \times 1,080$ pixels $)$; shutter speed, auto $(1 / 30 \sim 1 / 30,000 \mathrm{~s})$; aperture, auto (F1.2 360) (Fig. 2A).

\section{2) Assessment of dental plaque stained using a two-tone disclosing solution}

Dental plaque was stained with a two-tone disclosing solution and a photograph was obtained with the DSLR camera using the same method described in the previous section. DSLR photography used the following imaging parameters: resolution (image size), $(\mathrm{S} 2,902 \times 2,000$ pixels
[6 megabytes]); shutter speed, 1/160 s; aperture, F32; ISO, 200; white balance, auto; magnification, 1:3; photography mode, manual (Fig. 2B).

Stained dental plaque was scored by a calibrated examiner as follows: 0 for no staining, 1 for pink staining, and 2 for blue staining (Table 1). To obtain the scoring reliability for the evaluated area, re-evaluation was performed after 7 days.

\section{3) Assessment of dental plaque using the fluorescence image}

The RGB values for dental plaque on the fluorescence images were obtained using an image analysis program (Adobe Photoshop version 21.1.32; Adobe, Seoul, Korea). The marked points on the DSLR images were selected as the regions of interest for the RGB analysis. Dental caries and calculus were excluded, as they could affect the study results. The red/green $(\mathrm{R} / \mathrm{G})$ ratio was calculated from the obtained RGB values (Fig. 1).

Based on the fluorescence image analysis results, the red fluorescence characteristics were clinically divided into three groups. Dental plaque on the fluorescence image was scored by a calibrated examiner as follows: 0 for no red fluorescence, 1 for orange or light red fluorescence,

Table 1. Descriptions of the Dental Plaque Score on White and Fluorescence Images

\begin{tabular}{cll}
\hline Plaque score & \multicolumn{1}{c}{ White light image } & \multicolumn{1}{c}{ Fluorescence image } \\
\hline 0 & No stained & $\begin{array}{c}\text { No red fluorescence } \\
\text { Orange or light red } \\
\text { fluorescence }\end{array}$ \\
2 & Stained as blueish tone & $\begin{array}{c}\text { Definitely red } \\
\text { fluorescence }\end{array}$ \\
\hline
\end{tabular}
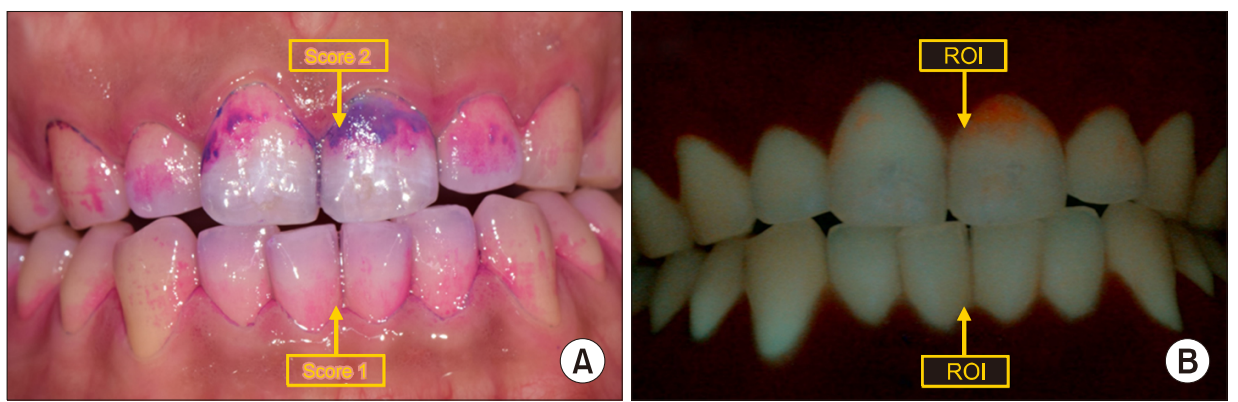

Fig. 2. Captured images. (A) Dental plaque stained with a two-tone disclosing solution and (B) red fluorescence image of dental plaque. Pinkish staining was assigned a score of 1 and bluish staining was assigned a score of 2. The scored area was selected as the region of interest (ROI) for analyzing the RGB values. 
and 2 for definitely red fluorescence (Table 1). To obtain the scoring reliability of the evaluated area, re-evaluation was performed after 7 days.

\section{Statistical analyses}

Before comparing the red fluorescence intensity of dental plaque in the fluorescence image according to dental plaque maturation, Cohen's kappa analysis was performed on the dental plaque evaluation results to obtain the reliability for the evaluation site. Excellent agreement of $0.90(p<0.001)$ was confirmed.

The relationship between dental plaque maturation and the $\mathrm{R} / \mathrm{G}$ ratio was evaluated using Spearman's rank correlation. In addition, differences in red fluorescence values according to dental plaque accumulation were evaluated using one-way analysis of variance followed by

Table 2. Red Fluorescence Values according to Dental Plaque Maturation and the Relationship between the Groups

\begin{tabular}{cccc}
\hline $\begin{array}{c}\text { Dental plaque } \\
\text { maturation }\end{array}$ & $\begin{array}{c}\text { Number of labial } \\
\text { surfaces }(\mathrm{n}=166)\end{array}$ & $\mathrm{R} / \mathrm{G}$ ratio & $\mathrm{r}^{\mathrm{c}}$ \\
\hline 0 & $49(29.5)$ & $0.78 \pm 0.13^{\mathrm{a}}$ & \\
1 & $67(40.4)$ & $1.26 \pm 0.29^{\mathrm{a}}$ & 0.907 \\
2 & $50(30.1)$ & $3.08 \pm 2.01^{\mathrm{b}}$ & \\
p-value & & $<0.001$ & $<0.001$ \\
\hline
\end{tabular}

Values are presented as number (\%) or mean \pm standard deviation. $\mathrm{R} / \mathrm{G}$ : red/green.

${ }^{\mathrm{a}, \mathrm{b}}$ Different letters within the same column indicate significant differences between the groups according to Scheffe's post-hoc analysis at $\mathrm{a}=0.05$. The $\mathrm{R} / \mathrm{G}$ values are the ratios of red pixels to green pixels in dental plaque images captured by the Q-ray cam. ${ }^{c} \mathrm{r}$ is the Spearman correlation coefficient.
Scheffe's post-hoc test to identify statistically significant differences between the groups. To analyze the agreement between plaque scores on the white-light images and fluorescence images, Cohen's kappa analysis was performed.

Statistical analysis was performed using IBM SPSS Statistics, version 21.0 (IBM Corp., Armonk, NY, USA) with a significance level of $5 \%$.

\section{Results}

\section{Relationship between dental plaque maturation and red fluorescence intensity}

A stronger intensity of red fluorescence was observed in QLF images wherein dental plaque was stained with a bluish tone (mature plaque) ( $\mathrm{p}<0.001$, Table 2$)$. In addition, an excellent positive correlation was observed between stained dental plaque and the intensity of red fluorescence $(\mathrm{p}<0.001$, Table 2$)$

\section{Possibility of a new plaque scoring system}

Table 1 shows the classification of the degree of dental plaque staining into three stages based on the common fluorescence pattern according to dental plaque accumulation. Analysis of the group means of the $\mathrm{R} / \mathrm{G}$ ratio according to the classified criteria confirmed significant differences between the groups $(\mathrm{p}<0.001$, Table 3$)$. In addition, a substantial agreement $(\mathrm{r}=0.637)$ was observed between the fluorescence plaque score and the plaque score obtained by staining with the two-tone solution $(\mathrm{p}<$ 0.001 , Table 3).

Table 3. Red Fluorescence Intensity according to Dental Plaque Maturation and Its Agreement with Dental Plaque Score Obtained by Staining with a Two-Tone Disclosing Solution

\begin{tabular}{ccccc}
\hline $\begin{array}{c}\text { Fluorescence } \\
\text { plaque score }\end{array}$ & $\begin{array}{c}\text { Number of labial } \\
\text { surfaces }(\mathrm{n}=166)\end{array}$ & $\mathrm{R} / \mathrm{G}$ ratio & $\mathrm{r}^{\mathrm{c}}$ & Agreement $^{\mathrm{d}}$ \\
\hline 0 & $49(29.5)$ & $0.85 \pm 0.23^{\mathrm{a}}$ & & 0.823 \\
1 & $60(36.1)$ & $1.19 \pm 0.32^{\mathrm{a}}$ & & 0.637 \\
2 & $57(34.3)$ & $2.86 \pm 1.97^{\mathrm{b}}$ & $<0.001$ & $<0.001$ \\
\hline p-value & & $<0.001$ & & \\
\hline
\end{tabular}

Values are presented as number (\%) or mean \pm standard deviation.

R/G: red/green.

a,b Different letters within the same column indicate significant differences between the groups according to Scheffe's post-hoc analysis at $\mathrm{a}=0.05$. The $\mathrm{R} / \mathrm{G}$ values are the ratios of red pixels to green pixels in dental plaque images captured by the Q-ray cam.

${ }^{c} \mathrm{r}$ is the Spearman correlation coefficient.

${ }^{\mathrm{d}}$ Agreement between stained plaque score and fluorescence plaque score. 


\section{Discussion}

The QLF technology can detect porphyrin, a bacterial metabolite in dental plaque, using $405 \mathrm{~nm}$ visible light ${ }^{12-14)}$. Based on this principle, the present study was conducted to analyze dental plaque maturation in the oral cavity from fluorescence images obtained using the QLF technology and to develop a new dental plaque index based on the results.

By analyzing the relationship between dental plaque maturation and the red fluorescence intensity observed in the QLF images, we attempted to confirm whether dental plaque stained with a two-tone disclosing solution was well reflected in the QLF image. An excellent correlation was observed between the variables, with a correlation coefficient of 0.907 (Table 2). This may be because the area with a high degree of plaque maturation (stained purple) had a significantly higher $\mathrm{R} / \mathrm{G}$ ratio in the fluorescence image when compared with the area with little or no plaque accumulation. Although there was no significant difference between the groups, the green value was higher than the red value in areas where dental plaque did not accumulate, showing an $\mathrm{R} / \mathrm{G}$ ratio of less than 1.0. On the other hand, the red value was higher than the green value in the $R / G$ ratio of areas with a plaque score of 1 (plaque stained with a pinkish tone) (Table 2). In this study, a two-tone dental staining agent was used as a standard to compare dental plaque maturation. The two-tone plaque-disclosing solution used in this study stained the "young" plaque in a pinkish tone "old" or "mature" plaque in a bluish tone. The pink dye adheres to all types of plaque, whereas the blue dye adheres and diffuses more easily into denser/thicker plaque. Hence, young plaque stains pink and the mature plaque stains blue-purple ${ }^{9,17)}$. According to this principle, it can be inferred that dental plaque stained with a bluish tone is pathogenic plaque that has not been removed from the oral cavity for at least 3 days $^{7,18)}$. It has been reported that dental plaque in the oral cavity becomes pathogenic with changes in the composition of the bacterial flora after 3 days of accumulation and shows red fluorescence in visible light of $405 \mathrm{~nm}^{14,19}$. Therefore, in case of bluish dental plaque that was accumulated in the oral cavity for a long time, red fluorescence was observed on the QLF image and an excellent correlation between stained dental plaque and the $\mathrm{R} / \mathrm{G}$ ratio was observed. Considering these results, it is clinically reasonable to propose a fluorescencebased plaque scoring system. On the other hand, while suggesting such a scoring system, factors such as dental calculus should be considered with respect to the pathogenicity of the accumulated dental plaque. In the present study, the red fluorescence of the stained plaque and that of dental plaque in the QLF image was compared and the results were presented without considering the influence of dental calculus. However, the potential pathogenicity of dental calculus should be considered, as it showed a strong red fluorescence pattern with an $\mathrm{R} / \mathrm{G}$ ratio of 10 to 172 depending on the size and volume of calculus (data not shown). The dental calculus forms by calcification of the plaque accumulated over a long period. Therefore, it is easier for dental plaque to accumulate in areas where calculus is present when compared with areas without calculus, which may lead to a higher incidence of periodontal disease ${ }^{20)}$. Therefore, it is necessary to develop a dental plaque index in the future that considers both dental plaque and calculus.

To overcome the limitations of the disclosing solution and to provide clinical convenience, studies have attempted to detect dental plaque using the QLF technology ${ }^{15,16)}$. Although a correlation was demonstrated between the degree of dental plaque maturation and the quantitative value analyzed using the QLF technique, it did not provide an intuitive classification standard required in the clinical field. In the present study, we attempted to confirm the possibility of developing a new dental plaque scoring system by dividing the common characteristics of fluorescence into different stages according to the degree of dental plaque accumulation and by comparing the $\mathrm{R} / \mathrm{G}$ ratio for each group with the existing plaque score (Table 1). No evidence of any red fluorescence or plaque accumulation on the QLF image was assigned a score of 0 . Dental plaque accumulated in a thin layer with orange-red fluorescence was assigned a score of 1. Dental plaque accumulated on the tooth surface as a thick layer with evident red fluorescence was assigned a score of 2 . Based on this classification, the level of dental plaque on the 
fluorescence image was reclassified and the R/G ratio was evaluated. Similar to the evaluation result of the stained dental plaque, dental plaque with an evident red fluorescence (score 2) showed a significant difference from that in the other groups. Nevertheless, the R/G ratio in the fluorescence images with a score of 1 or 2 was higher than that in images with plaque staining scores of 1 or 2 after staining with the disclosing solution. In addition, score 1 according to the existing method on some tooth surfaces was classified as score 2 on the QLF image. There are two possible explanations for this finding. 1) In case of the existing method (disclosing agent), there was a possibility of overestimation due to staining of debris such as the acquired pellicle or materia alba. On the other hand, the QLF image allows a more accurate evaluation by clearly distinguishing the pathogenic dental plaque. 2) It is possible that subgingival calculus near the gingival margin was not well observed in the white image, but became visible in the fluorescence image. A dental disclosing agent works on the principle of penetrating dental plaque and staining it $^{8,9)}$. However, there is a limit to distinguishing from DSLR images in calculus accumulated along the gingival margin because the calculus is not penetrated by the disclosing agent. However, in QLF images, even dental calculus exhibits stronger red fluorescence. Hence, this technology is expected to be more appropriate for the detection of dental plaque. Based on these results, it is necessary to consider the objectification of ambiguous standards including debris and dental calculus while developing a new dental biofilm scoring system in the future.

The demand for teledentistry is increasing with the development of modern society. Particularly, the coronavirus disease-2019 pandemic has recently created concerns about infection during the medical process and has changed the direction from direct face-to-face interaction between medical staff and patients to the use of telemedicine. Accordingly, the demand for telemedicine in dentistry is also increasing ${ }^{21,22}$. Thus, the results of the present study are expected to be a cornerstone in the field of clinical dental hygiene considering the demands of a changing society.

Despite the numerous advantages of dental plaque detection using the QLF technology, this study has several limitations. In case of subjects with malocclusion, it was difficult to accurately evaluate dental plaque using only the fluorescence images due to shading. In patients with irregular dentition, access of oral hygiene products to dental plaque is limited physically and chemically. Hence, the oral hygiene status is worse and the incidence of oral diseases is higher in subjects with malocclusion when compared with those having normal dentition ${ }^{23)}$. However, this limitation can be overcome by using portable QLF technology to increase the tooth surface accessibility of the device. Only the anterior teeth were included in the analysis to minimize factors that may affect the results. However, there may be a difference in the distribution of dental plaque and red fluorescence between posterior teeth and anterior teeth, as teeth have different thicknesses and structures according to the area. Therefore, it is necessary to analyze posterior teeth in the future. As a first step in developing a new dental plaque index, this study evaluated the clinical aspects of dental plaque in the fluorescence images according to its maturation level. Thus, we confirmed the possibility of developing a valid dental plaque index from the QLF fluorescence images. Future researches that involve a greater sample size and ensure the consistency and validity of the assessments made by the clinicians might help in proposing a future-oriented dental plaque index consistent with the direction required in the field of modern dental hygiene.

The present study was an introductory study to develop a fluorescence-based plaque scoring system. This study could distinguish the clinical characteristics of red fluorescence based on the degree of dental plaque maturation. Based on our results, we expect to develop a new and valid dental plaque scoring system that caters to the requirements of a changing era.

\section{Notes}

\section{Conflict of interest}

No potential conflict of interest relevant to this article was reported. 


\section{Ethical approval}

This study was approved by the Committee's Institutional Review Board of Suwon Science College (Approval number: IRB2-7008167-AB-N-01-201706-HR-003-01).

\section{Author contributions}

Conceptualization: Hye-Young Oh. Data collection and analysis: Hye-Young Oh and Eun-Ha Jung. Formal analysis: Eun-Ha Jung. Supervision: Hye-Young Oh. Writing - original draft: Eun-Ha Jung. Writing - review \& editing: Hye-Young Oh.

\section{ORCID}

Eun-Ha Jung, https://orcid.org/0000-0002-3737-5899

Hye-Young Oh, https://orcid.org/0000-0001-5848-1917

\section{References}

1. Marsh PD, Bradshaw DJ: Dental plaque as a biofilm. J Ind Microbiol 15: 169-175, 1995. https://doi.org/10.1007/BF01569822

2. Mariotti A: Dental plaque-induced gingival diseases. Ann Periodontol 4: 7-19, 1999. https://doi.org/10.1902/annals.1999.4.1.7

3. Listgarten MA: The role of dental plaque in gingivitis and periodontitis. J Clin Periodontol 15: 485-487, 1988. https://doi.org/10.1111/j.1600-051x.1988.tb01019.x

4. De David SC, Mário TG, De Freitas GC, Kantorski KZ, Wikesjö UME, Moreira CHC: Correlation between plaque control and gingival health using short and extended oral hygiene intervals. Clin Oral Investig 22: 2593-2597, 2018. https://doi.org/10.1007/s00784-018-2358-5

5. O'Leary TJ, Drake RB, Naylor JE: The plaque control record. J Periodontol 43: 38, 1972. https://doi.org/10.1902/jop.1972.43.1.38

6. Loe H, Silness J: Periodontal disease in pregnancy. I. Prevalence and severity. Acta Odontol Scand 21: 533-551, 1963. https://doi.org/10.3109/00016356309011240

7. Datta D, Kumar SR, Narayanan A, Bapilus L, Anandan S: Disclosing solutions used in dentistry. World J Pharm Res 6: 1648-1656, 2017.

8. Fasoulas A, Pavlidou E, Petridis D, Mantzorou M, Seroglou K, Giaginis C: Detection of dental plaque with disclosing agents in the context of preventive oral hygiene training programs. Heliyon 5: e02064, 2019. https://doi.org/10.1016/j.heliyon.2019.e02064

9. Block PL, Lobene RR, Derdivanis JP: A two-tone dye test for dental plaque. J Periodontol 43: 423-426, 1972. https://doi.org/10.1902/jop.1972.43.7.423

10. Jung IH, Yeon KH, Song HR, Hwang YS: Cytotoxicity of dental disclosing solution on gingival epithelial cells in vitro. Clin Exp Dent Res 6: 669-676, 2020. https://doi.org/10.1002/cre2.321

11. Han SY, Kim BR, Ko HY, Kwon HK, Kim BI: Assessing the use of Quantitative Light-induced Fluorescence-Digital as a clinical plaque assessment. Photodiagnosis Photodyn Ther 13: 34-39, 2016. https://doi.org/10.1016/j.pdpdt.2015.12.002

12. de Josselin de Jong E, Sundström F, Westerling H, Tranaeus S, ten Bosch JJ, Angmar-Månsson B: A new method for in vivo quantification of changes in initial enamel caries with laser fluorescence. Caries Res 29: 2-7, 1995. https://doi.org/10.1159/000262032

13. Kim BI: QLF concept and clinical implementation. J Korean Dent Assoc 49: 443-450, 2011.

14. Lee ES, Kang SM, Ko HY, Kwon HK, Kim BI: Association between the cariogenicity of a dental microcosm biofilm and its red fluorescence detected by Quantitative Light-induced Fluorescence-Digital (QLF-D). J Dent 41: 1264-1270, 2013. https://doi.org/10.1016/j.jdent.2013.08.021

15. Hwang HR, Cho YS, Kim BI: Assessment of clinical applicability of a new plaque scoring system using Quantitative Light-Induced Fluorescence-Digital. J Dent Hyg Sci 14: 150-157, 2014.

16. Kim M, Lee SY, Cho YS: Validity and reliability of the plaque score using Qraycam. J Dent Hyg Sci 15: 377-382, 2015. http://doi.org/10.17135/jdhs.2015.15.3.377

17. Gallagher IH, Fussell SJ, Cutress TW: Mechanism of action of a two-tone plaque disclosing agent. J Periodontol 48: 395-396, 1977. https://doi.org/10.1902/jop.1977.48.7.395

18. Chetruş V, Ion IR: Dental plaque- classification, formation, and identification. Int J Med Dent 3: 139-143, 2013.

19. Kim YS, Lee ES, Kwon HK, Kim BI: Monitoring the maturation process of a dental microcosm biofilm using the 
Quantitative Light-induced Fluorescence-Digital (QLF-D). J Dent 42: 691-696, 2014.

https://doi.org/10.1016/j.jdent.2014.03.006

20. White DJ: Dental calculus: recent insights into occurrence, formation, prevention, removal and oral health effects of supragingival and subgingival deposits. Eur J Oral Sci 105(5 Pt 2): 508-522, 1997.

https://doi.org/10.1111/j.1600-0722.1997.tb00238.x

21. Ghai S: Teledentistry during COVID-19 pandemic. Diabetes Metab Syndr 14: 933-935, 2020. https://doi.org/10.1016/j.dsx.2020.06.029

22. Rahman N, Nathwani S, Kandiah T: Teledentistry from a patient perspective during the coronavirus pandemic. $\mathrm{Br}$ Dent J 14: 1-4, 2020.

https://doi.org/10.1038/s41415-020-1919-6

23. Zotti F, Dalessandri D, Salgarello S, et al.: Usefulness of an app in improving oral hygiene compliance in adolescent orthodontic patients. Angle Orthod 86: 101-107, 2016.

https://doi.org/10.2319/010915-19.1 